\title{
History of cosmic evolution with modified Gauss-Bonnet-dilatonic coupled term
}

\author{
Subhra Debnath ${ }^{1, \mathrm{a}}$, Soumendra Nath Ruz ${ }^{2, \mathrm{~b}}$, Ranajit Mandal ${ }^{3, \mathrm{c}}$, Abhik Kumar Sanyal ${ }^{1, \mathrm{~d}}$ \\ ${ }^{1}$ Department of Physics, Jangipur College, Murshidabad 742213, India \\ 2 Department of Physics, Ramananda Centenary College, Purulia 723151, India \\ ${ }^{3}$ Department of Physics, University of Kalyani, Nadia 741235, India
}

Received: 16 August 2016 / Accepted: 28 April 2017 / Published online: 18 May 2017

(C) The Author(s) 2017. This article is an open access publication

\begin{abstract}
Gauss-Bonnet-dilatonic coupling in four dimensions plays an important role to explain late-time cosmic evolution. However, this term is an outcome of the low energy string effective action and thus ought to be important in the early universe too. Unfortunately, a phase-space formulation of such a theory does not exist in the literature due to branching. We therefore consider a modified theory of gravity, which contains a nonminimally coupled scalar-tensor sector in addition to a higher-order scalar curvature invariant term with Gauss-Bonnet-dilatonic coupling. Such an action unifies early inflation with late-time cosmic acceleration. The quantum version of the theory is also well behaved.
\end{abstract}

\section{Introduction}

A smooth luminosity-distance versus redshift curve reveals that distant supernovae appear dimmer than usual $[1,2]$. This issue may be explained by modifying the energy-momentum tensor appearing on the right hand side of Einstein's equation, giving rise to the so-called dark energy. Almost all the solutions to Einstein's equations result in accelerated expansion of the universe, if some form of dark energy is invoked. As a result, supernovae data, dark energy and latetime cosmic acceleration became synonyms. Nevertheless, a Friedmann-like matter dominated era $\left(a(t) \propto t^{\frac{2}{3}}, a(t)\right.$ being the scale factor), should be followed by such accelerated expansion, which is a recent phenomenon; otherwise, it would tell against the structures we observe. Further, the solution should also match other experimental data, e.g. the matter-radiation equality at redshift $z \approx 3200[3,4]$, decou-

\footnotetext{
a e-mail: subhra_dbnth@yahoo.com

b e-mail: ruzfromju@gmail.com

c e-mail: ranajitmandalphys@gmail.com

de-mail: sanyal_ak@yahoo.com
}

pling at $z \approx 1080$ [5] and anisotropy of CMBR, released from WMAP data [6]. These experimental data rule out some of the dark energy models. However, the problem with the remaining models is two-fold: first of all, the models are indistinguishable from each other, and second, complicated models with two of more scalar fields and sometimes with reverse sign of kinetic energy is required to exhibit crossing of the phantom divide line. In this sense, a better option is to modify the left hand side of Einstein's equation by incorporating higher-order curvature invariant terms in the Einstein-Hilbert action. Such a theory is dubbed a modified theory of gravity. A successful modified theory of gravity was first proposed by Nojiri and Odintsov $[7,8]$, in the form

$$
\begin{aligned}
A_{1} & =\int \mathrm{d}^{4} x \sqrt{-g} F(R) \\
& =\int \mathrm{d}^{4} x \sqrt{-g}\left[\frac{R}{16 \pi G}+\beta R^{2}+\gamma R^{-1}\right],
\end{aligned}
$$

where the $R^{2}$ term dominates at the very early universe, leading to inflation, $R$ dominates in the middle, so that nucleosynthesis, CMBR, structure formation etc. remain unaltered from standard model, and $R^{-1}$ dominates at the latestage of cosmic evolution, leading to late-time acceleration of the universe. A scalar mode called "scalaron" appears due to the scalar-tensor equivalence of higher-order theory. The mass of the scalar field may be adjusted, by suitably fine tuning $\beta$, so that the model passes solar test. Thus, a model which appears to reconcile early inflation with latetime cosmic acceleration satisfying all the presently available experimental data, bypassing the dark energy issue, is at hand. The only problem is: the $R^{-1}$ term is not recognized at all in any physical argument. The same has also been attempted later by Modak et al. [9] successfully, with the action 


$$
\begin{aligned}
A_{1} & =\int \mathrm{d}^{4} x \sqrt{-g} F(R) \\
& =\int \mathrm{d}^{4} x \sqrt{-g}\left[\frac{R}{16 \pi G}+\beta R^{2}+\gamma R^{\frac{3}{2}}\right],
\end{aligned}
$$

which explains reionization, in addition. Although $R^{\frac{3}{2}}$ is an artefact of Noether symmetry, none of the terms $\left(R^{-1}\right.$ or $\left.R^{\frac{3}{2}}\right)$ is generated by one-loop quantum gravitational corrections. Therefore, even the slightest presence of these terms in the early universe shatters all attempts to obtain a renormalized theory of gravity.

The attempt to modify gravity was initiated almost a century ago by Weyl [10], soon after the advent of the general theory of relativity (GTR). Later, it was realized that the inevitable gravitational collapse is due to the application of GTR beyond its domain of validity. However, the attempt to find a quantum counterpart at the Planck scale or beyond revealed that GTR suffers from ultraviolet divergence and so it is not renormalizable. A renormalized theory of gravity by incorporating curvature squared terms in the EinsteinHilbert action in the form

$A_{1}=\int \mathrm{d}^{4} x \sqrt{-g}\left[\frac{R}{16 \pi G}+\beta R^{2}+\gamma R_{\mu \nu} R^{\mu \nu}\right]$

was presented in the late twentieth century [11]. Unfortunately, this came at a very high price, since fourth-order derivatives lead to ghosts in the perturbation series about the linearized theory. An attempt to construct a second-order theory out of higher-order curvature invariant terms lead to a particular combination viz. the Gauss-Bonnet combination, $\mathcal{G}=R^{2}-4 \gamma R_{\mu \nu} R^{\mu \nu}+\gamma R_{\mu \nu \delta \gamma} R^{\mu \nu \delta \gamma}$. Although one may consider the (Wald) entropy effect of Gauss-Bonnet (GB) gravity in four dimensions [12-15], it is notable that the variation of GB Lagrangian is a total derivative in four dimensions, and therefore it does not contribute to the four dimensional field equations, as well as to the black hole solutions [16-19]. Thus, in order to study the contributions of GB term, solutions to Lanczos-Lovelock gravity [20,21], which is realized in five and even higher dimensions, are studied.

There exist a variety of important features of GB gravity. Its effects on the speed of graviton propagation together with the appearance of potentially super-luminal modes have been investigated $[22,23]$ and the problem with its unusual causal structure has also been resolved [24]. It was further applied to the investigation of a possible resolution of the initial singularity and graceful exit problem [25-27]. Stability criteria have also been studied [28]. In addition, the influences of GB gravity have been investigated as regards various physical phenomena, such as superconductors [29-32], hydrodynamics [33,34], LHC black holes [35], dark matter [36], dark energy $[37,38]$ and shear viscosity $[39,40]$.

If one therefore wants to restrict the study to four dimensions with Gauss-Bonnet term, it is possible with a Gauss-
Bonnet-dilatonic coupled term, which arises naturally as the leading order of the $\alpha^{\prime}$ expansion of heterotic superstring theory, where $\alpha^{\prime}$ is the inverse string tension [41-46]. Such a term works fairly well in four dimensions; e.g. it admits black-hole solutions [47-51] and gives rise to cosmic inflation at the early epoch [52]. Late-time accelerated expansion of the universe is particularly a very special feature of such an action [52-57]. Moreover, important issues like late-time dominance of dark energy after a scaling matter era, thus alleviating the coincidence problem crossing the phantom divide line, and compatibility with the observed spectrum of cosmic background radiation have also been addressed recently [5861]. It also gives fruitful results in a Noether symmetry study as well [62]. In a nutshell, the gravitational action containing Einstein-Gauss-Bonnet-dilatonic coupling has been able to explain the cosmological puzzle successfully.

Here, we therefore aim to expatiate the effect of EinsteinGauss-Bonnet-dilatonic coupled action in the very early universe, following canonical quantization. This primarily requires a canonical formulation of the theory. However, while performing a canonical analysis of the LanczosLovelock action [20,21] under a $3+1$ decomposition, Deser and Franklin noticed that the presence of cubic kinetic terms and quadratic constraints makes the theory intrinsically nonlinear [63]. The pathology associated with Lovelock AdS black branes and AdS/CFT was also discussed by Takahashi and Soda [64]. So one cannot perform a standard Hamiltonian formulation of such an action following the conventional Legendre transformation. As a result, diffeomorphic invariance is not manifest and the standard canonical formulation of the theory is obscure. Such a situation arises because the Lagrangian is quartic in the velocities, and therefore the expressions for velocities are multivalued functions of momentum, resulting in the so-called multiply branched Hamiltonian with cusps. This makes the classical solution unpredictable, as at any instant of time one can jump from one branch of the Hamiltonian to the other, as the equation of motion allows for such jumps. It is important to mention that, in principle, the particle always remains in one branch of the Hamiltonian and the presence of cusps restricts the domain of the variables of the problem. To make things more apparent, we use a toy model following $[65,66]$ :

$A=\int\left[\frac{1}{4} \dot{q}^{4}-\frac{1}{2} \alpha \dot{q}^{2}\right] \mathrm{d} t$,

for which

$\frac{\mathrm{d} p(\dot{q})}{\mathrm{d} t}=0 ; \quad p(\dot{q})=p_{0}=\dot{q}^{3}-\alpha \dot{q}$.

One is now required to solve $\dot{q}$ as a function of the integration constant $p_{0}$, which is possible uniquely for $\alpha<0$ as depicted in Fig. 1. However, for $\alpha>0$, the inverse image of the velocity $\dot{q}$ is single valued if the initial momentum 


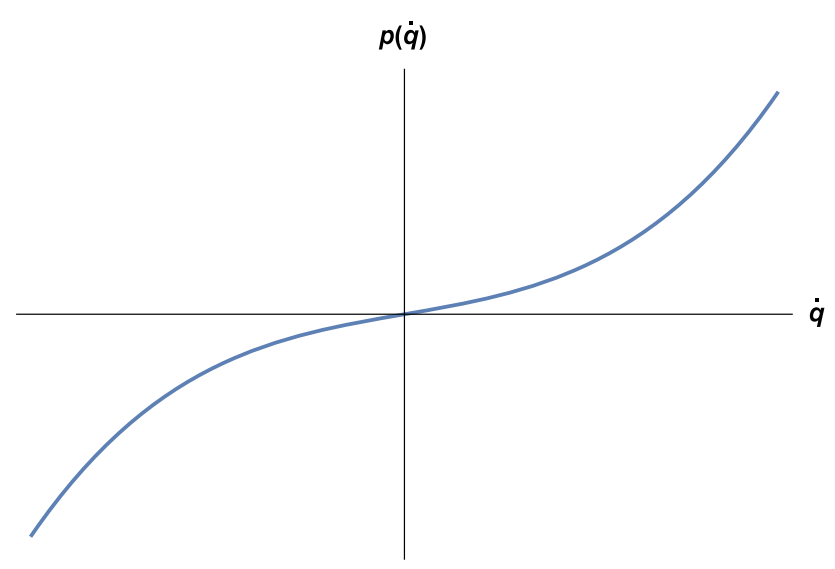

Fig. 1 For $\alpha<0$, Legendre map from $\dot{q}$ to $p$ shows one to one correspondence between the two

$\left(p_{0}\right)$ lies beyond the closed interval $\left[p_{1}, p_{2}\right]$; doubly valued, if the initial momenta is at one of the critical values $p_{1}$ or $p_{2}$, and finally within the interval, it takes three values, as depicted in Fig. 2. Therefore within the interval, for a particular value of $p$, one does not know which initial value of $\dot{q}$ it belongs to, and the equations of motion allows instantaneous switching (jumps) from one $\dot{q}$ value to another, since these instantaneous jumps leave $p$ unchanged and satisfy equation of motion. Further, since each given $p$ corresponds to one or three values of $\dot{q}$, the Hamiltonian

$H=\frac{3}{4} \dot{q}^{4}-\frac{\alpha}{2} \dot{q}^{2}$

is also mulltivalued with cusps, as shown in Fig. 3. At any instant of time one does not know which "branch" of the Hamiltonian to use, thus one may propagate for a while with one choice of the Hamiltonian, then switch to another and so on. Since the switching may be done after arbitrarily small time intervals, one may visualize the classical motion as a succession of zigzags which happen in an unpredictable manner. Thus, the behaviour of the system described by the action (4) remains unpredictable for a range of initial data of nonvanishing extent. Further, the momentum does not provide a complete set of commuting observables, resulting in nonunitary time evolution of quantum states.

Although Lanczos-Lovelock gravity $[20,21]$ shows unitary time evolution of quantum states, when expanded perturbatively about the flat Minkowski background, nonperturbatively the situation is miserable. In Sect. 2, we show that the Einstein-Gauss-Bonnet-dilatonic action suffers from the same disease. Presently, there is no standard technique to handle this issue. Two recent techniques in this fields are the Legendre-Fenchel transformation [66] and the generalized Legendre transformation [67]. However, it has been shown that, for the same system, the Hamiltonians obtained following the two techniques are not related through canonical transformation [68]. Thus, one does not

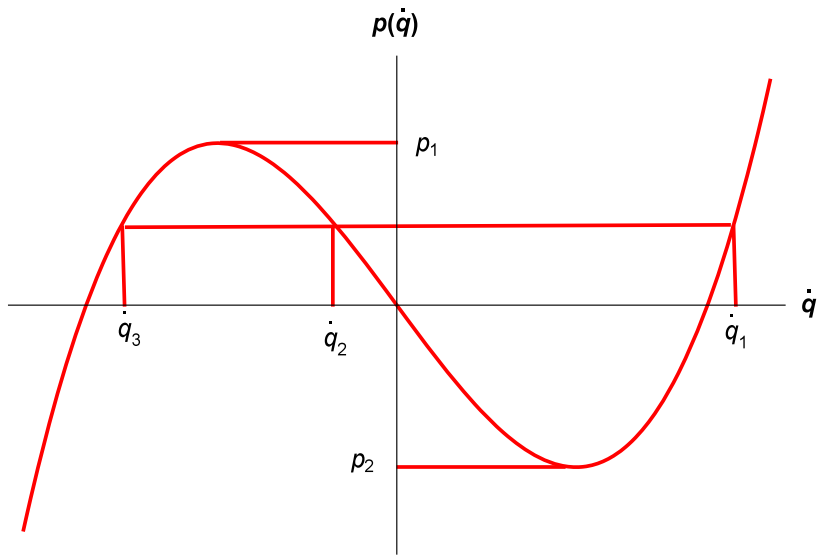

Fig. 2 For $\alpha>0$, Legendre map from $\dot{q}$ to $p$ shows multivaluedness of inverse images $\dot{q}$

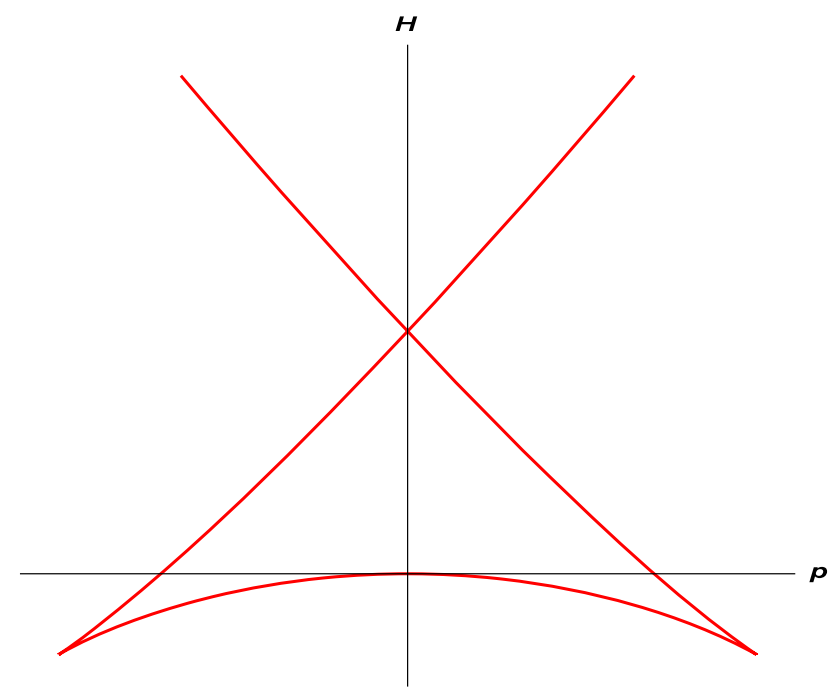

Fig. 3 Phase-space portrait depicts the multivaluedness of the Hamiltonian $H$ with cusps

know which one is the correct Hamiltonian. Nevertheless, one can bypass the issue of branching arising out of higher degrees, by incorporating higher-order terms [69]. This has been addressed recently, to alleviate the problem of branching in Lanczos-Lovelock gravity $[20,21]$, by modifying the action, associating with the case a scalar curvature invariant term [68]. Here in Sect. 3, we follow the same procedure in homogeneous and isotropic minisuperspace model, to cast the modified action

$$
\begin{aligned}
A= & \int \sqrt{-g} \mathrm{~d}^{4} x\left[\frac{R}{16 \pi G}+\xi(\phi)\left(\mathcal{G}+\beta R^{2}\right)\right. \\
& \left.-\frac{1}{2} \phi_{, \mu} \phi^{, \mu}-V(\phi)\right]
\end{aligned}
$$

in canonical ADM form [70]. The quantum description of the theory leads to a Schrödinger-like equation, where the proper volume acts as an internal time parameter. The effec- 
tive Hamiltonian operator has been found to be hermitian following the appropriate ordering prescription, for which a probabilistic interpretation is straightforward. The semiclassical approximation shows an oscillatory behaviour of the wave function about a classical inflationary solution. It therefore appears that the action (7) is a better option to demonstrate the evolutionary history of the universe.

\section{Problem in canonization of Gauss-Bonnet-dilatonic coupling}

As already mentioned, the Gauss-Bonnet-dilatonic coupled term appears as the leading order in low energy effective heterotic superstring theory. Although it can explain latetime cosmological evolution and fits other cosmological data when associated with the Einstein-Hilbert term, it contributes in the early universe as well. Therefore, it is important to study the quantum cosmological aspect of such a term. This requires a Hamiltonian formulation of the theory. In this section we aim to show that this is a non-trivial issue even in the homogeneous and isotropic minisuperspace model. The action we start with is

$$
\begin{aligned}
A_{1}= & \int \sqrt{-g} \mathrm{~d}^{4} x\left[\frac{R}{16 \pi G}+\xi(\phi) \mathcal{G}-\frac{1}{2} \phi_{, \mu} \phi^{, \mu}-V(\phi)\right] \\
& +\Sigma_{R}+\xi(\phi) \Sigma_{\mathcal{G}},
\end{aligned}
$$

where

$\Sigma_{R}=\frac{1}{8 \pi G} \oint_{\partial \mathcal{V}} K \sqrt{h} \mathrm{~d}^{3} x$,

$\Sigma_{\mathcal{G}}=4 \oint_{\partial \mathcal{V}}\left(2 G_{i j} K^{i j}+\frac{\mathcal{K}}{3}\right) \sqrt{h} \mathrm{~d}^{3} x$,

are the boundary terms corresponding to $R$ and $\mathcal{G}$, respectively. Here $\mathcal{G}$, is coupled with $\xi(\phi)$, and $V(\phi)$ is the dilatonic potential. The symbol $\mathcal{K}$ stands for $\mathcal{K}=\left(K^{3}-3 K K^{i j} K_{i j}+\right.$ $2 K^{i j} K_{i k} K_{j}^{k}$ ) where $K$ is the trace of the extrinsic curvature tensor $K_{i j}$, and $G_{i j}$ is the Einstein tensor built out of the induced metric $h_{i j}$ on the boundary. In the homogeneous and isotropic Robertson-Walker metric, viz.,

$$
\begin{aligned}
\mathrm{d} s^{2}= & -N(t)^{2} \mathrm{~d} t^{2}+a^{2}(t) \\
& \times\left[\frac{\mathrm{d} r^{2}}{1-k r^{2}}+r^{2}\left(\mathrm{~d} \theta^{2}+\sin ^{2} \theta \mathrm{d} \phi^{2}\right)\right],
\end{aligned}
$$

the expressions for $R$ and $\mathcal{G}$ are

$$
\begin{aligned}
& R=\frac{6}{N^{2}}\left(\frac{\ddot{a}}{a}+\frac{\dot{a}^{2}}{a^{2}}+N^{2} \frac{k}{a^{2}}-\frac{\dot{N} \dot{a}}{N a}\right), \\
& \mathcal{G}=\frac{24}{N^{3} a^{3}}(N \ddot{a}-\dot{N} \dot{a})\left(\frac{\dot{a}^{2}}{N^{2}}+k\right) .
\end{aligned}
$$

Let us first express the action (8) in terms of the basic variable, $h_{i j}=a^{2}=z$. This choice helps not only to take care of the boundary terms, but it also allows one to cast the action in ADM form as well $[71,72]$. In terms of the new variable, the action (8) reads

$$
\begin{aligned}
A_{1}= & \int\left[\frac{3 \sqrt{z}}{16 \pi G}\left(\frac{\ddot{z}}{N}-\frac{\dot{N} \dot{z}}{N^{2}}+2 k N\right)\right. \\
& +\frac{3 \xi(\phi)}{N \sqrt{z}}\left(\frac{\dot{z}^{2} \ddot{z}}{N^{2} z}+4 k \ddot{z}-\frac{\dot{z}^{4}}{2 N^{2} z^{2}}-\frac{\dot{N} \dot{z}^{3}}{N^{3} z}-\frac{2 k \dot{z}^{2}}{z}-\frac{4 k \dot{N} \dot{z}}{N}\right) \\
& \left.+z^{\frac{3}{2}}\left(\frac{\dot{\phi}^{2}}{2 N}-V N\right)\right] \mathrm{d} t+\Sigma_{R}+\xi(\phi) \Sigma_{\mathcal{G}} .
\end{aligned}
$$

Here

$\Sigma_{R}=-\frac{3 \sqrt{z} \dot{z}}{16 \pi G N}$

$\Sigma_{\mathcal{G}}=-\frac{\dot{z}}{N \sqrt{z}}\left(\frac{\dot{z}^{2}}{N^{2} z}+12 k\right)$.

Upon integration by parts, the total derivative terms get cancelled with the boundary term and the action (12) takes the form

$$
\begin{aligned}
A_{1}= & \int\left[\frac{1}{16 \pi G}\left(-\frac{3 \dot{z}^{2}}{2 N \sqrt{z}}+6 k N \sqrt{z}\right)\right. \\
& \left.-\frac{\xi^{\prime} \dot{z} \dot{\phi}}{N \sqrt{z}}\left(\frac{\dot{z}^{2}}{N^{2} z}+12 k\right)+z^{\frac{3}{2}}\left(\frac{1}{2 N} \dot{\phi}^{2}-V N\right)\right] \mathrm{d} t .
\end{aligned}
$$

The canonical momenta are

$$
\begin{aligned}
& p_{z}=-\frac{3 \dot{z}}{16 \pi G N \sqrt{z}}-\frac{3 \xi^{\prime} \dot{\phi}}{N \sqrt{z}}\left(\frac{\dot{z}^{2}}{N^{2} z}+4 k\right), \\
& p_{\phi}=-\frac{\xi^{\prime} \dot{z}}{N \sqrt{z}}\left(\frac{\dot{z}^{2}}{N^{2} z}+12 k\right)+\frac{z^{\frac{3}{2}} \dot{\phi}}{N}, \\
& p_{N}=0 .
\end{aligned}
$$

The $N$ variation equation,

$$
\begin{aligned}
- & \frac{3}{16 \pi G}\left(\frac{\dot{z}^{2}}{2 N^{2} \sqrt{z}}+2 k \sqrt{z}\right) \\
& -\frac{3 \xi^{\prime} \dot{z} \dot{\phi}}{N^{2} \sqrt{z}}\left(\frac{\dot{z}^{2}}{N^{2} z}+4 k\right)+z^{\frac{3}{2}}\left(\frac{1}{2 N^{2}} \dot{\phi}^{2}+V\right)=0,
\end{aligned}
$$

leads to the Hamiltonian constraint equation, which is essentially the $\left(\begin{array}{l}0 \\ 0\end{array}\right)$ equation of Einstein, viz. 


$$
\begin{aligned}
H_{c} & =N\left[-\frac{3}{16 \pi G}\left(\frac{\dot{z}^{2}}{2 N^{2} \sqrt{z}}+2 k \sqrt{z}\right)\right. \\
& \left.-\frac{3 \xi^{\prime} \dot{z} \dot{\phi}}{N^{2} \sqrt{z}}\left(\frac{\dot{z}^{2}}{N^{2} z}+4 k\right)+z^{\frac{3}{2}}\left(\frac{1}{2 N^{2}} \dot{\phi}^{2}+V\right)\right]=0 .
\end{aligned}
$$

The definitions of the momenta imply that they are up to third degree equations in $\dot{z}$ and so are not invertible in general. Therefore, the action (12) suffers from the problem of branching as discussed in the introduction. Hence, it is impossible to cast the Hamiltonian in canonical form and, despite diffeomorphic invariance of the theory, it is not possible to express the Hamiltonian as $H_{c}=N \mathcal{H}$.

\section{Modified dilatonic coupled Gauss-Bonnet action}

A host of techniques are presently available in the literature to get rid of the above awesome situation, as already cited. Although these techniques alleviate the problem of branching, no two phase-space Hamiltonians are related through a canonical transformation, and therefore no one knows which is the correct description of the theory under consideration. However, the situation is considerably improved bypassing the associated problem, by incorporating an additional higher-order curvature invariant term in the action, as already noticed earlier [68,69]. Under an appropriate field redefinition followed by conformal transformation, it is always possible reduce the string frame action (to the first order in $\alpha^{\prime}$ ) to the form in Einstein's frame given in (7). Such a field redefinition is usually performed to get rid of higher-order derivatives from the field equations. Here our purpose is to demonstrate the fact that the scalar curvature squared term can alleviate the problem of branching. Therefore, we consider an additional $R^{2}$ term in the form given in (8). Including appropriate boundary terms, such an action reads

$$
\begin{aligned}
A_{2}= & \int \sqrt{-g} \mathrm{~d}^{4} x\left[\frac{R}{16 \pi G}+\xi(\phi)\left(\beta R^{2}+\mathcal{G}\right)-\frac{1}{2} \phi_{, \mu} \phi^{, \mu}\right. \\
& -V(\phi)]+\Sigma_{R}+\xi(\phi)\left[\Sigma_{\mathcal{G}}+\beta\left(\Sigma_{R_{1}^{2}}+\Sigma_{R_{2}^{2}}\right)\right], \quad(18)
\end{aligned}
$$

where

$$
\begin{aligned}
\Sigma_{R^{2}}= & \oint_{\partial \mathcal{V}}{ }^{4} R K \sqrt{h} \mathrm{~d}^{3} x=\Sigma_{R_{1}^{2}}+\Sigma_{R_{2}^{2}}=\oint_{\partial \mathcal{V}}{ }^{3} R K \sqrt{h} \mathrm{~d}^{3} x \\
& +\oint_{\partial \mathcal{V}}\left({ }^{4} R-{ }^{3} R\right) K \sqrt{h} \mathrm{~d}^{3} x
\end{aligned}
$$

${ }^{3} R$ being the Ricci scalar in 3 -space. In the RobertsonWalker minisuperspace (10) under consideration,

$$
\Sigma_{R_{1}^{2}}=-36 k \frac{\dot{z}}{N \sqrt{z}} \text { and } \Sigma_{R_{2}^{2}}=-18 \frac{\dot{z}}{N^{3} \sqrt{z}}\left(\ddot{z}-\frac{\dot{N} \dot{z}}{N}\right) \text {. }
$$

The reason for splitting the boundary term in two parts has been discussed in detail earlier [71-73]. Now upon integration by parts, some of the total derivative terms are removed from the action (18) and one is left with

$$
\begin{aligned}
A_{2}= & \int\left[\frac{1}{16 \pi G}\left(-\frac{3 \dot{z}^{2}}{2 N \sqrt{z}}+6 k N \sqrt{z}\right)\right. \\
& +\frac{9 \xi \beta}{\sqrt{z}}\left(\frac{\ddot{z}^{2}}{N^{3}}-\frac{2 \dot{N} \ddot{z} \ddot{z}}{N^{4}}+\frac{\dot{N}^{2} \dot{z}^{2}}{N^{5}}+\frac{2 k \dot{z}^{2}}{N z}+4 k^{2} N\right) \\
& -\frac{36 \beta k \xi^{\prime} \dot{z} \dot{\phi}}{N \sqrt{z}}-\frac{\xi^{\prime} \dot{z} \dot{\phi}}{N \sqrt{z}}\left(\frac{\dot{z}^{2}}{N^{2} z}+12 k\right) \\
& \left.+z^{\frac{3}{2}}\left(\frac{1}{2 N} \dot{\phi}^{2}-V N\right)\right] \mathrm{d} t+\xi(\phi) \beta \Sigma_{R_{2}^{2}} .
\end{aligned}
$$

At this stage, following Horowitz [74], let us introduce an auxiliary variable

$$
Q=\frac{\partial A}{\partial \ddot{z}}=\frac{18 \xi \beta}{N^{3} \sqrt{z}}\left(\ddot{z}-\frac{\dot{N} \dot{z}}{N}\right)
$$

judiciously in the action (21), thus:

$$
\begin{aligned}
A_{2}= & \int\left[\frac{1}{16 \pi G}\left(-\frac{3 \dot{z}^{2}}{2 N \sqrt{z}}+6 k N \sqrt{z}\right)+Q \ddot{z}-\frac{\dot{N}}{N} \dot{z} Q\right. \\
& -\frac{N^{3} \sqrt{z}}{36 \xi \beta} Q^{2}+\frac{18 \xi \beta k}{\sqrt{z}}\left(\frac{\dot{z}^{2}}{N z}+2 k N\right) \\
& -\frac{36 \beta k \xi^{\prime} \dot{z} \dot{\phi}}{N \sqrt{z}}-\frac{\xi^{\prime} \dot{z} \dot{\phi}}{N \sqrt{z}}\left(\frac{\dot{z}^{2}}{N^{2} z}+12 k\right) \\
& \left.+z^{\frac{3}{2}}\left(\frac{1}{2 N} \dot{\phi}^{2}-V N\right)\right] \mathrm{d} t+\xi(\phi) \beta \Sigma_{R_{2}^{2} .}
\end{aligned}
$$

Upon integration by parts, the rest of the boundary terms gets cancelled with the total derivative term, and the action (23) is finally expressed as

$$
\begin{aligned}
A_{2}= & \int\left[\frac{1}{16 \pi G}\left(-\frac{3 \dot{z}^{2}}{2 N \sqrt{z}}+6 k N \sqrt{z}\right)-\dot{Q} \dot{z}-\frac{\dot{N}}{N} \dot{z} Q\right. \\
& -\frac{N^{3} \sqrt{z}}{36 \beta \xi} Q^{2}+\frac{18 \beta \xi k}{\sqrt{z}}\left(\frac{\dot{z}^{2}}{N z}+2 k N\right) \\
& -\frac{36 \beta k \xi^{\prime} \dot{z} \dot{\phi}}{N \sqrt{z}}-\frac{\xi^{\prime} \dot{z} \dot{\phi}}{N \sqrt{z}}\left(\frac{\dot{z}^{2}}{N^{2} z}+12 k\right) \\
& \left.+z^{\frac{3}{2}}\left(\frac{1}{2 N} \dot{\phi}^{2}-V N\right)\right] \mathrm{d} t .
\end{aligned}
$$

The canonical momenta are

$$
\begin{aligned}
p_{Q}= & -\dot{z} \\
p_{z}= & -\frac{3 \dot{z}}{16 \pi G N \sqrt{z}}-\dot{Q}-\frac{Q \dot{N}}{N}+\frac{36 \beta k \xi \dot{z}}{N z^{\frac{3}{2}}}-\frac{36 \beta k \xi^{\prime} \dot{\phi}}{N \sqrt{z}} \\
& -\frac{3 \xi^{\prime} \dot{\phi}}{N \sqrt{z}}\left(\frac{\dot{z}^{2}}{N^{2} z}+4 k\right) \\
p_{\phi}= & -\frac{36 \beta k \xi^{\prime} \dot{z}}{N \sqrt{z}}-\frac{\xi^{\prime} \dot{z}}{N \sqrt{z}}\left(\frac{\dot{z}^{2}}{N^{2} z}+12 k\right)+\frac{z^{\frac{3}{2}}}{N} \dot{\phi} \\
p_{N}= & -\frac{Q \dot{z}}{N} .
\end{aligned}
$$


The $N$ variation equation reads

$$
\begin{aligned}
& -\frac{3}{16 \pi G}\left(\frac{\dot{z}^{2}}{2 N^{2} \sqrt{z}}+2 k \sqrt{z}\right)-\frac{Q \ddot{z}}{N}-\frac{\dot{Q} \dot{z}}{N}+\frac{N^{2} \sqrt{z}}{12 \beta \xi} Q^{2} \\
& +\frac{18 \xi \beta k}{\sqrt{z}}\left(\frac{\dot{z}^{2}}{N^{2} z}-2 k\right)-\frac{36 \beta k \xi^{\prime} \dot{z} \dot{\phi}}{N^{2} \sqrt{z}}-\frac{3 \xi^{\prime} \dot{z} \dot{\phi}}{N^{2} \sqrt{z}}\left(\frac{\dot{z}^{2}}{N^{2} z}+4 k\right) \\
& +z^{\frac{3}{2}}\left(\frac{1}{2 N^{2}} \dot{\phi}^{2}+V\right)=0 .
\end{aligned}
$$

The action (24) is singular due to the diffeomorphic invariance. This must be reflected in the $N$ variation equation, since it should not contain a second derivative term. The presence of the second derivative term $(\ddot{z})$ indicates that the situation is altogether different from GTR. This awful situation may be handled easily without going through Dirac's constraint analysis. This is possible because, in view of the definition of $Q$ given in (22), the $\ddot{z}$ term may be removed from the above $N$ variation equation (26), which now takes the form

$$
\begin{aligned}
& -\frac{3}{16 \pi G}\left(\frac{\dot{z}^{2}}{2 N^{2} \sqrt{z}}+2 k \sqrt{z}\right)-\frac{\dot{z} \dot{Q}}{N}-\frac{\dot{N} \dot{z} Q}{N^{2}}+\frac{N^{2} \sqrt{z}}{36 \beta \xi} Q^{2} \\
& +\frac{18 \xi \beta k}{\sqrt{z}}\left(\frac{\dot{z}^{2}}{N^{2} z}-2 k\right)-\frac{36 \beta k \xi^{\prime} \dot{z} \dot{\phi}}{N^{2} \sqrt{z}}-\frac{3 \xi^{\prime} \dot{z} \dot{\phi}}{N^{2} \sqrt{z}}\left(\frac{\dot{z}^{2}}{N^{2} z}+4 k\right) \\
& +z^{\frac{3}{2}}\left(\frac{1}{2 N^{2}} \dot{\phi}^{2}+V\right)=0 .
\end{aligned}
$$

Now, since Eq. (27) does not contain a second derivative term, it should be treated as a constraint of the system. It can easily be verified that this is the Hamiltonian of the system in disguise. We can therefore write the Hamiltonian constraint equation

$$
\begin{aligned}
H_{c}= & N\left[-\frac{3}{16 \pi G}\left(\frac{\dot{z}^{2}}{2 N^{2} \sqrt{z}}+2 k \sqrt{z}\right)-\frac{\dot{z} \dot{Q}}{N}-\frac{\dot{N} \dot{z} Q}{N^{2}}\right. \\
& +\frac{N^{2} \sqrt{z}}{36 \beta \xi} Q^{2}+\frac{18 \xi \beta k}{\sqrt{z}}\left(\frac{\dot{z}^{2}}{N^{2} z}-2 k\right)-\frac{36 \beta k \xi^{\prime} \dot{z} \dot{\phi}}{N^{2} \sqrt{z}} \\
& \left.-\frac{3 \xi^{\prime} \dot{z} \dot{\phi}}{N^{2} \sqrt{z}}\left(\frac{\dot{z}^{2}}{N^{2} z}+4 k\right)+z^{\frac{3}{2}}\left(\frac{1}{2 N^{2}} \dot{\phi}^{2}+V\right)\right]=0,
\end{aligned}
$$

which is constrained to vanish. Now, using the expression

$$
\begin{aligned}
p_{Q} p_{z}= & \frac{3 \dot{z}^{2}}{16 \pi G N \sqrt{z}}+\dot{z} \dot{Q}+\frac{\dot{N}}{N} \dot{z} Q-\frac{36 k \beta \xi \dot{z}^{2}}{N z^{\frac{3}{2}}} \\
& +\frac{36 \beta k \xi^{\prime} \dot{z} \dot{\phi}}{N \sqrt{z}}+\frac{3 \dot{z} \xi^{\prime} \dot{\phi}}{N \sqrt{z}}\left(\frac{\dot{z}^{2}}{N^{2} z}+4 k\right)
\end{aligned}
$$

and also using the definitions of momenta (25a, 25c), the Hamiltonian constraint equation in terms of the phase space variables is expressed as

$$
\begin{aligned}
& H_{c}=\frac{3}{16 \pi G}\left(\frac{p_{Q}{ }^{2}}{2 N \sqrt{z}}-2 k N \sqrt{z}\right)-p_{Q} p_{z}+\frac{N^{3} Q^{2} \sqrt{z}}{36 \beta \xi} \\
& -\frac{18 \xi k \beta}{\sqrt{z}}\left(\frac{p_{Q}^{2}}{N z}+2 k N\right)+\frac{N p_{\phi}^{2}}{2 z^{\frac{3}{2}}}+\frac{\xi^{\prime 2} p_{Q}{ }^{6}}{2 N^{5} z^{\frac{9}{2}}}+\frac{648 k^{2} \beta^{2} \xi^{\prime 2} p_{Q}^{2}}{N z^{\frac{5}{2}}} \\
& +\frac{72 k^{2} \xi^{\prime 2} p_{Q}{ }^{2}}{N z^{\frac{5}{2}}}-\frac{36 \beta k \xi^{\prime} p_{Q} p_{\phi}}{z^{2}}+\frac{12 k \xi^{\prime 2} p_{Q}}{N^{3} z^{\frac{7}{2}}}-\frac{\xi^{\prime} p_{Q}^{3} p_{\phi}}{N^{2} z^{3}} \\
& -\frac{12 k \xi^{\prime} p_{Q} p_{\phi}}{z^{2}}+\frac{36 \beta k \xi^{\prime 2} p_{Q}^{4}}{N^{3} z^{\frac{7}{2}}}+\frac{432 \beta k^{2} \xi^{\prime 2} p_{Q}^{2}}{N z^{\frac{5}{2}}} \\
& +N V z^{\frac{3}{2}}=0 .
\end{aligned}
$$

In the above form of the phase-space Hamiltonian (30), various momenta appear in products whose powers are at least of second order and reach up to sixth order $\left(p_{Q}{ }^{6}\right)$. Of course, this is very inconvenient in order to form the operators. Even if one does, a large number of initial (boundary) conditions are required to solve the quantum counterpart, which are not available. Furthermore, the Hamiltonian (30) also contains cross terms in higher degree of the momenta $\left(p_{Q}{ }^{3} p_{\phi}\right)$, which makes things even more complicated. On the contrary, one can observe that the configuration variable $Q$ appears only quadratically in (30). So, in order to handle such an awful situation, it is suggestive to express the above Hamiltonian in terms of the basic variables $\left\{K_{i j}, \pi^{i j}\right\}$. This is possible under the canonical transformation from $\left\{Q, p_{Q}\right\}$ to $\left\{x, p_{x}\right\}: Q=\frac{p_{x}}{N}$ and $p_{Q}=-\dot{z}=-N x$. Hence, the Hamiltonian in terms of the basic variables reads

$$
\begin{aligned}
H_{c}= & N\left[x p_{z}+\frac{p_{x}^{2} \sqrt{z}}{36 \beta \xi}+\frac{p_{\phi}^{2}}{2 z^{\frac{3}{2}}}+\frac{\xi^{\prime}}{z^{2}}\left(36 \beta k x+\frac{x^{3}}{z}+12 k x\right) p_{\phi}\right. \\
& +\frac{3}{16 \pi G}\left(\frac{x^{2}}{2 \sqrt{z}}-2 k \sqrt{z}\right)-\frac{18 \xi k \beta}{\sqrt{z}}\left(\frac{x^{2}}{z}+2 k\right) \\
& +\frac{\xi^{\prime 2} x^{6}}{2 z^{\frac{9}{2}}}+\frac{648 k^{2} \beta^{2} \xi^{\prime 2} x^{2}}{z^{\frac{5}{2}}}+\frac{72 k^{2} \xi^{\prime 2} x^{2}}{z^{\frac{5}{2}}}+\frac{12 k \xi^{\prime 2} x^{4}}{z^{\frac{7}{2}}} \\
& \left.+\frac{36 \beta k \xi^{\prime 2} x^{4}}{z^{\frac{7}{2}}}+\frac{432 \beta k^{2} \xi^{\prime 2} x^{2}}{z^{\frac{5}{2}}}+V z^{\frac{3}{2}}\right]=N \mathcal{H}=0 . \quad \text { (31) }
\end{aligned}
$$

The fact that Eq. (31) gives the correct Hamiltonian description of the theory (18) under consideration has been established in the appendix. The above description of the phasespace Hamiltonian explores the very importance of basic variables $\left\{h_{i j}, p_{i j} ; K_{i j}, \pi^{i j}\right\}$, which are $\left\{z, p_{z} ; x, p_{x}\right\}$, respectively, in the minisuperspace under consideration. First of all, the diffeomorphic invariance $H_{c}=N \mathcal{H}$, being an artefact of general covariance, is manifest in GTR. In higher-order theory of gravity, as one can observe, it is manifest only when the Hamiltonian is expressed in terms of the basic phase-space variables. Arbitrary phase-space variables, even if those are related to the basic variables under a canonical transformation, are no good. Next, the above Hamilton constraint equation (31) is only quadratic 
in the momenta, and there are no cross terms. Thus, it is now convenient to canonically quantize the Hamilton constraint equation (31). Moreover, the momentum $p_{z}$ appears only linearly, which is nice if we are to realize the internal variable $z$ as time co-ordinate, upon quantization.

One may wonder why basic variables were not used from the very beginning. The answer to the question is: if the action were expressed in terms of $\left\{h_{i j}, K_{i j}\right\}$ from the very beginning, the Hessian determinant would have vanished and the Lagrangian would have been singular. Such a situation may be dealt with Dirac's constraint analysis [75]. Nevertheless, Dirac's analysis implicitly assumes that $h_{i j}$ and $K_{i j}$ should be kept fixed at the boundary. As a result, supplementary boundary terms are not required. In the process, one loses the cherished Gibbons-Hawking-York (GHY) boundary term [76,77], which is associated with the entropy of a black hole. Even in the weak field approximation $(\xi \rightarrow 0)$, it cannot be retrieved. On the contrary, it has been shown that the supplementary boundary term associated with the higher-order theory of gravity reproduces the expected ADM energy [70] upon passing to the Hamiltonian formalism, and the correct expression of the entropy of a Schwarzschild black hole may be found in the semiclassical limit [78]. In the present analysis therefore, along with $h_{i j}$, the Ricci scalar $R$ has been kept fixed at the boundary, and the action is supplemented by appropriate boundary terms. An auxiliary variable has been introduced so that the Lagrangian is non-singular. In this context, we also mention that the two techniques yield different phase-space Hamiltonians in general, as has been demonstrated recently [69].

It is now straightforward to write the action in the ADM canonical form:

$$
\begin{aligned}
A_{2} & =\int\left(\dot{z} p_{z}+\dot{x} p_{x}+\dot{\phi} p_{\phi}-N \mathcal{H}\right) \mathrm{d} t \mathrm{~d}^{3} x \\
& =\int\left(\dot{h}_{i j} p^{i j}+\dot{K}_{i j} \pi^{i j}+\dot{\phi} p_{\phi}-N \mathcal{H}\right) \mathrm{d} t \mathrm{~d}^{3} x .
\end{aligned}
$$

Thus, the problem of branching, which appeared due to the presence of a higher degree term in the action (8) has been bypassed by the introduction of a higher-order curvature invariant term- $R^{2}$. Note that such a term appears in the weak energy limit of all different quantum theories of gravity, and so the action (18) is a natural generalization of action (8). In fact, any higher-order curvature invariant term can cure the problem associated with a higher degree, but as $R_{\mu \nu} R^{\mu \nu}$ leads to a ghost, it is safe to handle the situation, with a scalar curvature invariant term.

\subsection{Canonical quantization}

The quantum version of Hamiltonian (31) is

$$
\begin{aligned}
& \frac{i \hbar}{\sqrt{z}} \frac{\partial \Psi}{\partial z}=-\frac{\hbar^{2}}{36 \beta \xi x}\left(\frac{\partial^{2}}{\partial x^{2}}+\frac{n}{x} \frac{\partial}{\partial x}\right) \Psi-\frac{\hbar^{2}}{2 x z^{2}} \frac{\partial^{2} \Psi}{\partial \phi^{2}} \\
& \quad+\frac{1}{z^{\frac{5}{2}}}\left(\frac{x^{2}}{z}+36 \beta k+12 k\right) \widehat{\xi^{\prime}} \widehat{p}_{\phi}+\frac{3}{16 \pi G}\left(\frac{x}{2 z}-\frac{2 k}{x}\right) \Psi \\
& \quad-\frac{18 k \beta \xi}{z}\left(\frac{x}{z}+\frac{2 k}{x}\right) \Psi+\frac{\widehat{\xi}^{\prime} x}{z^{3}}\left(\frac{x^{4}}{2 z^{2}}+\frac{12 k x^{2}}{z}+72 k^{2}\right. \\
& \left.\quad+648 k^{2} \beta^{2}+\frac{36 \beta k x^{2}}{z}+432 k^{2} \beta\right) \Psi+\frac{V z}{x} \Psi \\
& \quad=\hat{H}_{e} \Psi,
\end{aligned}
$$

where $n$ is the operator ordering index. The operator form of $\widehat{\xi}^{\prime} \widehat{p}_{\phi}$ appearing in the third term on the right hand side, may be inserted appropriately, only after knowing the specific form of $\xi(\phi)$, as this term also requires ordering. The specific form of $\xi(\phi)$ is also required to investigate the behaviour of the quantum theory, in a certain appropriate semiclassical approximation. Specific forms of $\xi(\phi)$ and $V(\phi)$ may be obtained if we invoke slow-roll inflation, so that an almost scale-invariant perturbation on large scales is successfully generated from quantum fluctuations of $\phi$. In view of the action (24), the $\left(\begin{array}{l}0 \\ 0\end{array}\right)$ equation of Einstein [(which is the essentially the Hamiltonian constraint equations (28), (30) or (31)] and the $\phi$ variation equation may be expressed under the standard gauge choice $N=1$, and setting $k=0$, in terms of the scale factor $a$ as

$$
\begin{aligned}
\frac{\dot{a}^{2}}{a^{2}}= & -96 \beta \xi \pi G\left[2 \frac{\dot{a} \dddot{a}}{a^{2}}-\frac{\ddot{a}^{2}}{a^{2}}+2 \frac{\dot{a}^{2} \ddot{a}}{a^{3}}-3 \frac{\dot{a}^{4}}{a^{4}}\right] \\
& -192 \pi G \beta \xi^{\prime} \dot{\phi}\left(\frac{\dot{a} \ddot{a}}{a^{2}}+\frac{\dot{a}^{3}}{a^{3}}\right)-64 \pi G \xi^{\prime} \dot{\phi}\left(\frac{\dot{a}^{3}}{a^{3}}\right) \\
& +\frac{8 \pi G}{3}\left(\frac{\dot{\phi}^{2}}{2}+V\right)
\end{aligned}
$$

and

$$
\begin{aligned}
& -24 \xi^{\prime} \dot{a}^{2} \ddot{a}-36 \beta \xi^{\prime} a \ddot{a}^{2}-72 \beta \xi^{\prime} \dot{a}^{2} \ddot{a} \\
& -36 \beta \xi^{\prime} \frac{\dot{a}^{4}}{a}+3 a^{2} \dot{a} \dot{\phi}+a^{3}\left(\ddot{\phi}+V^{\prime}\right)=0,
\end{aligned}
$$

respectively. Equations (34) and (35) may further be rearranged as

$$
\begin{aligned}
M_{\mathrm{pl}}^{2} H^{2}= & \frac{1}{3}\left(\frac{1}{2} \dot{\phi}^{2}+V\right)-12 \beta \xi\left[4 H^{4}\left(1+\frac{\dot{H}}{H^{2}}\right)\right. \\
& +4 H^{2} \dot{H}\left(1+\frac{\dot{H}}{H^{2}}\right)+2 H^{3}\left(\frac{\ddot{H}}{H^{2}}-2 \frac{\dot{H}^{2}}{H^{3}}\right)
\end{aligned}
$$




$$
\begin{aligned}
& \left.-H^{4}\left(1+\frac{\dot{H}}{H^{2}}\right)^{2}-3 H^{4}\right] \\
& -48 \beta \dot{\xi} H^{3}-8 \dot{\xi} H^{3}-24 H \dot{\xi} \dot{H},
\end{aligned}
$$

$$
\begin{aligned}
\ddot{\phi}+3 H \dot{\phi}= & -V^{\prime}+(6 \beta+1) 24 \xi^{\prime} H^{4} \\
& +(6 \beta+1) 24 \xi^{\prime} H^{2} \dot{H}+36 \beta \xi^{\prime} \dot{H}^{2}
\end{aligned}
$$

where $H \equiv \frac{\dot{a}}{a}$ denotes the expansion rate and $M_{\mathrm{pl}}^{2}=\frac{1}{8 \pi G}$. Since the GB coupling is a function of $\phi$, one has $\dot{\xi}=\xi^{\prime} \dot{\phi}$ and $\ddot{\xi}=\xi^{\prime \prime} \dot{\phi}^{2}+\xi^{\prime} \ddot{\phi}$. Now, due to the presence of an additional degree of freedom $\xi(\phi)$, along with the standard slowroll conditions of minimally coupled single-field inflation, viz. $\dot{\phi}^{2} \ll V$ and $|\ddot{\phi}| \ll 3 H|\dot{\phi}|$, one is required to impose two additional conditions, viz. $4|\dot{\xi}| H \ll 1$ and $|\ddot{\xi}| \ll|\dot{\xi}| H$ [79]. Instead of standard slow-roll parameters, it is customary to introduce a combined hierarchy of Hubble and GaussBonnet flow parameters [80-83]. First, the background evolution is described by a set of horizon flow functions (the behaviour of Hubble distance during inflation) starting from

$\epsilon_{0}=\frac{d_{H}}{d_{H_{i}}}, \quad$ where $d_{H}=H^{-1}$

is the Hubble distance, also called the horizon in our chosen units. Now a hierarchy of functions is defined in a systematic way as

$\epsilon_{l+1}=\frac{\mathrm{d} \ln \left|\epsilon_{l}\right|}{\mathrm{d} N}, \quad l \geq 0$.

In view of the definition $N=\ln \frac{a}{a_{i}}$, which implies $\dot{N}=H$, one can compute $\epsilon_{1}=\frac{\mathrm{d} \ln \mathrm{d}_{H}}{d N}$, which is the logarithmic change of Hubble distance per e-fold expansion $N$, and is the first slow-roll parameter $\epsilon_{1}=\dot{d}_{H}=-\frac{\dot{H}}{H^{2}}$. The above hierarchy allows one to compute $\epsilon_{2}=\frac{\mathrm{d} \ln \epsilon_{1}}{\mathrm{~d} N}=\frac{1}{H} \frac{\dot{\epsilon}_{1}}{\epsilon_{1}}$, which implies $\epsilon_{1} \epsilon_{2}=d_{H} \ddot{d}_{H}=-\frac{1}{H^{2}}\left(\frac{\ddot{H}}{H}-2 \frac{\dot{H}^{2}}{H^{2}}\right)$. In the same manner higher slow-roll parameters may be computed. Equation (39) essentially defines a flow in space with cosmic time being the evolution parameter, which is described by the equation of motion

$\epsilon_{0} \dot{\epsilon}_{l}-\frac{1}{d_{H_{i}}} \epsilon_{l} \epsilon_{l-1}=0, \quad l \geq 0$.

One can also check that (40) yields all the results obtained from the hierarchy defined in (39), using the definition (38). As already mentioned, the additional degree of freedom appearing due to the Gauss-Bonnet-dilatonic coupling requires one to introduce yet another hierarchy of GaussBonnet flow parameters:

$\delta_{1}=4 \dot{\xi} H \ll 1, \quad \delta_{i+1}=\frac{d \ln \left|\delta_{i}\right|}{d \ln a}, \quad$ with,$i \geq 1$.

Clearly for $i=1, \delta_{2}=\frac{d \ln \left|\delta_{1}\right|}{d N}=\frac{1}{\delta_{1}} \frac{\dot{\delta}_{1}}{N}$, and $\delta_{1} \delta_{2}=\frac{4}{H}(\ddot{\xi} H+$ $\dot{\xi} \dot{H})$ and so on. The slow-roll conditions therefore read $\left|\epsilon_{i}\right| \ll 1$ and $\left|\delta_{i}\right| \ll 1$, which is analogous to the standard slow-roll approximation, and Eqs. (36) and (37) may therefore be expressed as

$$
\begin{aligned}
M_{\mathrm{pl}}^{2} H^{2}= & \frac{1}{3}\left(\frac{1}{2} \dot{\phi}^{2}+V\right)-12 \beta \xi\left[3 H^{4}\left(1-\epsilon_{1}\right)^{2}\right. \\
& +2 H^{3} \overbrace{\left(1-\epsilon_{1}\right)}^{i}-3 H^{4}]-\left(2 H^{2}+12 \beta H^{2}\right)\left(1+\delta_{1}\right) \\
& +6 \beta H^{2}\left(1+\delta_{1} \epsilon_{1}\right)+\left(2 H^{2}+6 \beta H^{2}\right)
\end{aligned}
$$

and

$$
\begin{aligned}
\ddot{\phi} & +3 H \dot{\phi}=-V^{\prime}+\frac{1}{\dot{\phi}}\left[\left(6 H^{3}(6 \beta+1)\left(1+\delta_{1}\right)\right.\right. \\
& \left.-6 H^{3}(6 \beta+1)\left(1+\delta_{1} \epsilon_{1}\right)+9 H^{3} \beta\left(1+\delta_{1}\right)\left(1-\epsilon_{1}\right)^{2}\right] \\
& +\frac{9 \beta H^{3}}{\dot{\phi}}\left(\left(1+\delta_{1}\right)-\left(1-\epsilon_{1}\right)^{2}-1\right),
\end{aligned}
$$

respectively. In view of the slow-roll parameters, Eqs. (42), and (43) may be approximated to

$H^{2} \simeq \frac{1}{3 M_{\mathrm{pl}}^{2}} V$

$H \dot{\phi} \simeq-\frac{1}{3} V \mathcal{Q}$

where $\mathcal{Q}=\frac{V^{\prime}}{V}$. In deriving Eq. (45), the approximation arrived at in Eq. (44) has been used. The number of e-folds may then be computed as usual in view of the following relation:

$N(\phi) \simeq \int_{t}^{t_{f}} H \mathrm{~d} t=\int_{\phi}^{\phi_{f}} \frac{H}{\dot{\phi}} \mathrm{d} \phi \simeq \frac{1}{M_{\mathrm{pl}}^{2}} \int_{\phi_{f}}^{\phi} \frac{\mathrm{d} \phi}{\mathcal{Q}}$,

where $\phi$ and $\phi_{f}$ denote the values of the scalar field at the beginning $(t)$ and the end $\left(t_{f}\right)$ of inflation respectively. Let us now consider a specific model with a monomial potential and an inverse monomial GB coupling,

$V(\phi)=V_{1}+V_{0} \phi^{m}, \quad \xi(\phi)=\xi_{0} \phi^{-m}$

where $V_{0}, V_{1}, \xi_{0}$ and $m$ are constants. Under the choice $m=$ 2, $\mathcal{Q}$ may be expressed as

$\mathcal{Q}=\frac{V^{\prime}}{V}=\frac{2 V_{0} \phi}{V_{1}+V_{0} \phi^{2}}$.

Therefore the number of e-foldings (46) reads

$N(\phi)=\frac{1}{2 M_{\mathrm{pl}}^{2}}\left[\frac{V_{1}}{V_{0}} \ln \left(\frac{\phi}{\phi_{f}}\right)+\frac{\left(\phi^{2}-\phi_{f}^{2}\right)}{2}\right]$.

Now if we set $\frac{V_{1}}{V_{0}} \approx-6 M_{\mathrm{pl}}^{2}$, then $N(\phi) \gtrsim 60$, for $\phi \gtrsim 16.4 M_{\mathrm{pl}}$ and $\phi_{f} \sim 3.26 M_{\mathrm{pl}}$, which solves the horizon and flatness problems. Slow roll ends under the condition $\epsilon_{1}=\frac{M_{\mathrm{pl}}^{2}}{2}\left(\frac{V^{\prime}}{V}\right)^{2}=\frac{2 M_{\mathrm{pl}}^{2} \phi^{2}}{\left(\frac{V_{1}}{V_{0}}+\phi^{2}\right)^{2}} \geqslant 1$, which requires $\phi_{f} \lesssim 3.26 M_{\mathrm{pl}}$. Cosmological perturbation with dilatonic 
Gauss-Bonnet gravity has been studied extensively in the literature [84-87]. The presence of an additional $R^{2}$ term does not seem to alter the qualitative behaviour. At present, let us therefore concentrate on the results in the quantum domain, which is our main concern. Under the above choice of $m=2$ and $k=0$, the quantum equation (33) may now be expressed as

$$
\begin{aligned}
\frac{i \hbar}{\sqrt{z}} \frac{\partial \Psi}{\partial z}= & -\frac{\hbar^{2}}{36 \beta \xi x}\left(\frac{\partial^{2}}{\partial x^{2}}+\frac{n}{x} \frac{\partial}{\partial x}\right) \Psi-\frac{\hbar^{2}}{2 x z^{2}} \frac{\partial^{2} \Psi}{\partial \phi^{2}} \\
& -i \hbar \frac{\xi_{0} x^{2}}{z^{\frac{7}{2}}}\left(\frac{3 \Psi}{\phi^{4}}-\frac{2}{\phi^{3}} \frac{\partial \Psi}{\partial \phi}\right) \\
& +\left[\frac{3 x}{32 \pi G z}+\frac{2 \xi_{0}^{2} x^{5}}{\phi^{6} z^{5}}+\frac{V z}{x}\right] \Psi,
\end{aligned}
$$

where a Weyl symmetric ordering has been performed in the third term appearing on the right hand side. Now, again under a further change of variable, the above modified Wheelerde-Witt equation takes the look of the Schrödinger equation, viz.,

$$
\begin{aligned}
i \hbar \frac{\partial \Psi}{\partial \alpha}= & -\frac{\hbar^{2}}{54 \beta \xi}\left(\frac{1}{x} \frac{\partial^{2}}{\partial x^{2}}+\frac{n}{x^{2}} \frac{\partial}{\partial x}\right) \Psi-\frac{\hbar^{2}}{3 x \alpha^{\frac{4}{3}}} \frac{\partial^{2} \Psi}{\partial \phi^{2}} \\
& -i \hbar \frac{2 \xi_{0} x^{2}}{3 \alpha^{\frac{7}{3}}}\left(\frac{3 \Psi}{\phi^{4}}-\frac{2}{\phi^{3}} \frac{\partial \Psi}{\partial \phi}\right)+V_{e} \Psi=\hat{H}_{e} \Psi
\end{aligned}
$$

where $\alpha=z^{\frac{3}{2}}=a^{3}$ plays the role of internal time parameter. In the above, the effective potential $V_{e}$ is given by

$$
V_{e}=\frac{x}{16 \pi G \alpha^{\frac{2}{3}}}+\frac{4 \xi_{0}^{2} x^{5}}{3 \phi^{6} \alpha^{\frac{10}{3}}}+\frac{2 V \alpha^{\frac{2}{3}}}{3 x} \text {. }
$$

The hermiticity of $\hat{H}_{e}$ should enable one to write the continuity equation, which requires one to find $\frac{\partial \rho}{\partial \alpha}$, where $\rho=\Psi^{*} \Psi$. A little algebra leads to the following equation:

$$
\begin{aligned}
\frac{\partial \rho}{\partial \alpha}= & -\frac{\partial}{\partial x}\left[\frac{i \hbar}{54 \beta \xi x}\left(\Psi \Psi_{, x}^{*}-\Psi^{*} \Psi_{, x}\right)\right] \\
& -\frac{\partial}{\partial \phi}\left[\frac{i \hbar}{3 \alpha^{\frac{4}{3}} x}\left(\Psi \Psi_{, \phi}^{*}-\Psi^{*} \Psi_{, \phi}\right)-\frac{4 \xi_{0} x^{2}}{3 \alpha^{\frac{7}{3}} \phi^{3}} \Psi^{*} \Psi\right] \\
& +\frac{(n+1)}{x^{2}}\left(\Psi \Psi_{, x}^{*}-\Psi^{*} \Psi_{, x}\right) .
\end{aligned}
$$

Clearly, the continuity equation can be written, only under the choice $n=-1$, as

$\frac{\partial \rho}{\partial \alpha}+\nabla . \mathbf{J}=0$.

In the above, $\rho=\Psi^{*} \Psi$ and $\mathbf{J}=\left(\mathbf{J}_{x}, \mathbf{J}_{\phi}, 0\right)$ are the probability density and the current density, respectively, where

$$
\begin{aligned}
& \mathbf{J}_{x}=\frac{i \hbar}{54 \beta \xi x}\left(\Psi \Psi_{, x}^{*}-\Psi^{*} \Psi_{, x}\right), \\
& \mathbf{J}_{\phi}=\frac{i \hbar}{3 x \alpha^{\frac{4}{3}}}\left(\Psi \Psi_{, \phi}^{*}-\Psi^{*} \Psi_{, \phi}\right)-\frac{4 \xi_{0} x^{2}}{3 \alpha^{\frac{7}{3}} \phi^{3}} \Psi^{*} \Psi .
\end{aligned}
$$

In the process, the operator ordering index has been fixed as $n=-1$ from physical arguments. As already mentioned, here the variable $\alpha$ plays the role of internal time parameter. It is important to note that a standard quantum mechanical probabilistic interpretation of the theory has been possible taking $\alpha=z^{\frac{3}{2}}=a^{3}$, i.e. taking the proper volume as the internal time parameter.

\subsection{Classical and semiclassical solutions (under WKB approximation)}

To check the viability of the quantum equation (51) so obtained, one is required to test its behaviour under a certain appropriate semiclassical approximation. A semiclassical approximation should be performed with the full quantum equation, without assuming slow-roll conditions. This requires a viable classical solution of the field equations (36) and (37). Under the choice $V=V_{1}+V_{0} \phi^{2}, \xi=\xi_{0} \phi^{-2}$, where $V_{1}$ is a constant, the full classical field equations admit an exponential inflationary solution in the form

$a=a_{0} \mathrm{e}^{H t} \quad \phi=\phi_{0} \mathrm{e}^{-H t}$,

restricting the constants to $V_{1}=\frac{3 H^{2}}{8 \pi G}, \beta=-\frac{1}{6}$ and $V_{0}=-\frac{H^{2}}{2}$, where $H$ is yet another constant. Now, let us express Eq. (50) as

$$
\begin{gathered}
-\frac{\hbar^{2} \sqrt{z}}{36 \beta \xi x}\left(\frac{\partial^{2}}{\partial x^{2}}+\frac{n}{x} \frac{\partial}{\partial x}\right) \Psi-\frac{\hbar^{2}}{2 x z^{\frac{3}{2}}} \frac{\partial^{2} \Psi}{\partial \phi^{2}} \\
-i \hbar \frac{\partial \Psi}{\partial z}+i \hbar \frac{2 \xi_{0} x^{2}}{z^{3} \phi^{3}} \frac{\partial \Psi}{\partial \phi}+\mathcal{V} \Psi=0,
\end{gathered}
$$

where

$$
\mathcal{V}=\frac{3 x}{32 \pi G \sqrt{z}}+\frac{2 \xi_{0}^{2} x^{5}}{\phi^{6} z^{\frac{9}{2}}}-\frac{3 i \hbar \xi_{0} x^{2}}{z^{3} \phi^{4}}+\frac{V_{0} \phi^{2} z^{\frac{3}{2}}}{x}+\frac{V_{1} z^{\frac{3}{2}}}{x}
$$

The above equation may be treated as a time independent Schrödinger equation with the three variables, $x, z$ and $\varphi$, therefore, as usual, let us seek the solution of Eq. (57),

$\psi=\psi_{0} \mathrm{e}^{\frac{i}{\hbar} S(x, z, \phi)}$

and expand $S$ in a power series of $\hbar$ as

$S=S_{0}(x, z, \phi)+\hbar S_{1}(x, z, \phi)+\hbar^{2} S_{2}(x, z, \phi)+\cdots$.

Now inserting Eqs. (59) and (60) in Eq. (57) and equating the coefficients of different powers of $\hbar$ to zero, one obtains the following set of equations (up to second order): 


$$
\begin{aligned}
& \frac{\sqrt{z}}{36 \beta \xi x} S_{0, x}^{2}+\frac{S_{0, \phi}^{2}}{2 x z^{\frac{3}{2}}}+S_{0, z}-\frac{2 \xi_{0} x^{2}}{z^{3} \phi^{3}} S_{0, \phi}+\frac{3 x}{32 \pi G \sqrt{z}} \\
& +\frac{2 \xi_{0}^{2} x^{5}}{\phi^{6} z^{\frac{9}{2}}}+\frac{V_{0} \phi^{2} z^{\frac{3}{2}}}{x}+\frac{V_{1} z^{\frac{3}{2}}}{x}=0, \\
& -\frac{i \sqrt{z}}{36 \beta \xi x} S_{0, x x}-\frac{i n \sqrt{z}}{36 \beta \xi x^{2}} S_{0, x}-\frac{i S_{0, \phi \phi}}{2 x z^{\frac{3}{2}}}+S_{1, z} \\
& +\frac{\sqrt{z} S_{0, x} S_{1, x}}{18 \beta \xi x}+\frac{2 S_{0, \phi} S_{1, \phi}}{x z^{\frac{3}{2}}}+\frac{\xi_{0} x^{2}}{z^{3} \phi^{3}} S_{1, \phi}-\frac{3 i \xi_{0} x^{2}}{z^{3} \phi^{4}}=0, \\
& -i \frac{\sqrt{z} S_{1, x x}}{36 \beta \xi x}+\frac{\sqrt{z} S_{1, x}{ }^{2}}{36 \beta \xi x}+\frac{\sqrt{z} S_{0, x} S_{2, x}}{18 \beta \xi x}-i \frac{n \sqrt{z} S_{1, x}}{36 \beta \xi x^{2}}-i \frac{S_{1, \phi \phi}}{2 x z^{\frac{3}{2}}} \\
& +\frac{S_{1, \phi}}{2 x z^{\frac{3}{2}}}+\frac{2 S_{0, \phi} S_{2, \phi}}{x z^{\frac{3}{2}}}+S_{2, z}-\frac{\xi_{0} x^{2}}{z^{3} \phi^{3}} S_{2, \phi}=0,
\end{aligned}
$$

which are to be solved successively to find $S_{0}(x, z, \phi)$, $S_{1}(x, z, \phi)$ and $S_{2}(x, z, \phi)$ and so on. Now identifying $S_{0, x}$ as $p_{x} ; S_{0, z}$ as $p_{z}$ and $S_{0, \phi}$ as $p_{\phi}$ one can recover the classical Hamiltonian constraint equation $H_{c}=0$ (for $k=0$ ), given in Eq. (31) from Eq. (61a). Thus, $S_{0}(x, z)$ can now be expressed as

$S_{0}=\int p_{z} \mathrm{~d} z+\int p_{x} \mathrm{~d} x+\int p_{\phi} \mathrm{d} \phi$

apart from a constant of integration which may be absorbed in $\psi_{0}$. Clearly, $S_{0}$ may only be found, evaluating the above integral, which is impossible due to tight coupling. We therefore use the classical solution (56) for the purpose, and we relate the (once) independent variables $a$ and $\phi$ through their temporal function as $z=a^{2}=\frac{\left(a_{0} \phi_{0}\right)^{2}}{\phi^{2}}$, which is always possible (remember that, to find the trajectory of a parabola, we relate the co-ordinates $x$ and $y$, through their time dependence). The integrals in the above expression can now be evaluated using the definition of $p_{z}$ given in (25b), $p_{\phi}$ in (25c) and $p_{x}=N Q$, and also recalling the expression for $Q$ given in (22). Further, we choose $n=-1$, since the probability interpretation holds only for such a value of $n$. Hence, $x(=\dot{z}), \phi$ and the expressions of $p_{x}, p_{z} p_{\phi}$ can be expressed in terms of $z$ and $\phi$ as

$$
\begin{aligned}
x & =2 \mathrm{H} z, \quad \phi=\frac{a_{0} \phi_{0}}{\sqrt{z}}, \\
p_{x} & =-\frac{12 \xi_{0} \mathrm{H}^{2} z^{\frac{3}{2}}}{\left(a_{0} \phi_{0}\right)^{2}}=-\frac{3 \xi_{0} \sqrt{2 \mathrm{H}} x^{\frac{3}{2}}}{\left(a_{0} \phi_{0}\right)^{2}}, \\
p_{z} & =-\frac{3}{8 \pi G} \sqrt{z} \mathrm{H}+\frac{12 \xi_{0} \mathrm{H}^{3} z^{\frac{3}{2}}}{\left(a_{0} \phi_{0}\right)^{2}}, \\
p_{\phi} & =16 \xi_{0} \mathrm{H}^{3} \frac{\left(a_{0} \phi_{0}\right)^{3}}{\phi^{6}}-\mathrm{H} \frac{\left(a_{0} \phi_{0}\right)^{3}}{\phi^{2}},
\end{aligned}
$$

and the integrals in (62) are evaluated as

$$
\begin{aligned}
\int p_{x} \mathrm{~d} x & =-\frac{6 \xi_{0} \sqrt{2 \mathrm{H}} x^{\frac{5}{2}}}{5\left(a_{0} \phi_{0}\right)^{2}}=-\frac{48 \xi_{0} \mathrm{H}^{3} z^{\frac{5}{2}}}{5\left(a_{0} \phi_{0}\right)^{2}} \\
\int p_{z} \mathrm{~d} z & =-\frac{1}{4 \pi G} \mathrm{H} z^{\frac{3}{2}}+\frac{24 \xi_{0} \mathrm{H}^{3} z^{\frac{5}{2}}}{5\left(a_{0} \phi_{0}\right)^{2}} \\
\int p_{\phi} \mathrm{d} \phi & =-\frac{16 \xi_{0} \mathrm{H}^{3}\left(a_{0} \phi_{0}\right)^{3}}{5 \phi^{5}}+\mathrm{H} \frac{\left(a_{0} \phi_{0}\right)^{3}}{\phi}
\end{aligned}
$$

Finally, the explicit form of $S_{0}$ can be written

$$
\begin{aligned}
S_{0}= & -\frac{6 \xi_{0} \sqrt{2 \mathrm{H}} x^{\frac{5}{2}}}{5\left(a_{0} \phi_{0}\right)^{2}}-\frac{1}{4 \pi G} \mathrm{H} z^{\frac{3}{2}}+\frac{24 \xi_{0} \mathrm{H}^{3} z^{\frac{5}{2}}}{5\left(a_{0} \phi_{0}\right)^{2}} \\
& -\frac{16 \xi_{0} \mathrm{H}^{3}\left(a_{0} \phi_{0}\right)^{3}}{5 \phi^{5}}+\mathrm{H} \frac{\left(a_{0} \phi_{0}\right)^{3}}{\phi} \\
= & -\frac{1}{4 \pi G} \mathrm{H} z^{\frac{3}{2}}-\frac{8 \xi_{0} \mathrm{H}^{3} z^{\frac{5}{2}}}{\left(a_{0} \phi_{0}\right)^{2}}+\mathrm{H}\left(a_{0} \phi_{0}\right)^{2} \sqrt{z},
\end{aligned}
$$

where in the last expression, we have flipped $\phi$ to $z$, using the expression $\phi=\frac{a_{0} \phi_{0}}{\sqrt{z}}$, to express $S_{0}$ in terms of $z$. It may be mentioned that Eq. (61a) has never been solved for $S_{0}$, since it is extremely difficult, if not impossible. We have rather found $S_{0}$ using the classical solutions (56). So, for consistency, one can trivially check that the expression for $S_{0}(65)$ so obtained satisfies Eq. (61a) identically. In fact it should, because Eq. (61a) coincides with Hamiltonian constraint equation (31) for $k=0$. Moreover, one can also compute the zeroth-order on-shell action. Using the classical solution (56) one may express all the variables in terms of $t$ and substituting in the action (23), one obtains

$$
\begin{aligned}
A_{2}= & \int\left[-\frac{3}{4 \pi G} a_{0}^{3} \mathrm{H}^{2} \mathrm{e}^{3 \mathrm{H} t}-\frac{40 \xi_{0} a_{0}{ }^{3} \mathrm{H}^{4}}{\phi_{0}{ }^{2}} \mathrm{e}^{5 \mathrm{H} t}\right. \\
& \left.+a_{0}{ }^{3} \mathrm{H}^{2} \phi_{0}{ }^{2} \mathrm{e}^{\mathrm{H} t}\right] \mathrm{d} t .
\end{aligned}
$$

On integration, one thus finds

$$
\begin{gathered}
-\frac{1}{4 \pi G} a_{0}{ }^{3} \mathrm{He}^{3 \mathrm{H} t}-\frac{8 \xi_{0} a_{0}{ }^{3} \mathrm{H}^{3}}{\phi_{0}{ }^{2}} \mathrm{e}^{5 \mathrm{H} t}+a_{0}{ }^{3} \mathrm{H} \phi_{0}{ }^{2} \mathrm{e}^{\mathrm{H} t} \\
=-\frac{1}{4 \pi G} \mathrm{H} z^{\frac{3}{2}}-\frac{8 \xi_{0} \mathrm{H}^{3} z^{\frac{5}{2}}}{\left(a_{0} \phi_{0}\right)^{2}}+\mathrm{H}\left(a_{0} \phi_{0}\right)^{2} \sqrt{z}
\end{gathered}
$$

which is the same expression as Eq. (65). Therefore up to zeroth-order approximation, the wave function reads

$\psi=\psi_{0} \mathrm{e}^{\frac{i}{\hbar}\left[-\frac{1}{4 \pi G} \mathrm{H} z^{\frac{3}{2}}-\frac{8 \xi_{0} \mathrm{H}^{3} z^{\frac{5}{2}}}{\left(a_{0} \phi_{0}\right)^{2}}+\mathrm{H}\left(a_{0} \phi_{0}\right)^{2} \sqrt{z}\right]}$. 


\subsubsection{First-order approximation}

Now for $n=-1$, Eq. (61b) can be expressed as

$$
\begin{gathered}
-\frac{\sqrt{z}}{36 \beta \xi x}\left(i S_{0, x x}-2 S_{0, x} S_{1, x}-\frac{i}{x} S_{0, x}\right) \\
-\frac{1}{2 x z^{\frac{3}{2}}}\left(i S_{0, \phi \phi}-4 S_{0, \phi} S_{1, \phi}\right) \\
+S_{1, z}+\frac{\xi_{0} x^{2}}{z^{3} \phi^{3}} S_{1, \phi}-\frac{3 i \xi_{0} x^{2}}{z^{3} \phi^{4}}=0 .
\end{gathered}
$$

Using the expression for $S_{0}$ obtained in (65), one can express $S_{1, z}$ in view of the above equation as

$S_{1, z}=-\frac{i\left[\frac{6 \xi_{0} \mathrm{H}^{2}}{\left(a_{0} \phi_{0}\right)^{4}} z-\frac{3}{8} \frac{1}{z}\right]}{1-\frac{\left(a_{0} \phi_{0}\right)^{3}}{z^{\frac{3}{2}}}+\frac{16}{5} \frac{\xi_{0} \mathrm{H}^{2}}{a_{0} \phi_{0}} \sqrt{z}-\frac{4 \xi_{0} \mathrm{H}^{2}}{\left(a_{0} \phi_{0}\right)^{2}} z}$.

In principle, one can integrate to obtain $S_{1}$ in the form

$S_{1}=-i f(z)$.

Therefore, the wave function to first-order approximation reads

$\psi=\psi_{01} \mathrm{e}^{\frac{i}{\hbar}\left[-\frac{\mathrm{H}}{4 \pi G} z^{\frac{3}{2}}-\frac{8 \xi_{0} \mathrm{H}^{3}}{\left(a_{0} \phi_{0}\right)^{2}} z^{\frac{5}{2}}+\mathrm{H}\left(a_{0} \phi_{0}\right)^{2} \sqrt{z}\right]}$,

where

$\psi_{01}=\psi_{0} \mathrm{e}^{f(z)}$.

Thus, a first-order approximation only modifies the prefactor, keeping the oscillatory behaviour of the wave function intact. Since the wave function is oscillatory about the classical inflationary solution, the correspondence between the quantum equation and the classical equations has been established.

\section{Concluding remarks}

The Einstein-Gauss-Bonnet-dilatonic coupled action successfully explains the late-time accelerated expansion of the universe, fitting all the observed cosmological data fairly well. However, such a term appears in the weak energy limit of heterotic string theory and so contributes at the early stage of cosmological evolution as well. Hence, it is important to study the quantum dynamics. Unfortunately, the Hamiltonian structure of such an action does not exist, due to the problem of branching. We have associated an additional scalar curvature squared $\left(R^{2}\right)$ term with dilatonic coupling in the action, to bypass the issue of branching. It is important to mention that alleviation of the issue of branching by the introduction of higher-order curvature invariant term is not an artefact of the minisuperspace approach, in which many degrees of freedom are suppressed. Note that $R^{2}$ itself contains terms with higher degree, e.g. $\dot{a}^{4}$, in Robertson-Walker minisuperspace. The canonical formulation of $\alpha R+\beta R^{2}$ action has been presented by Boulware [88] in the whole superspace, which did not encounter the issue of branching, even in the presence of momentum constraints. However, since Boulware's approach is limited for a particular form of action, it is not possible to follow the approach for the more general action under present consideration. To start with, we have considered the simplest homogeneous and isotropic minisuperspace model. A viable quantum version of the action (18) has been presented, where the effective Hamiltonian is hermitian and a standard quantum mechanical probabilistic interpretation holds. The semiclassical approximation yields a wave function which shows oscillatory behaviour about a classical inflationary solution. Therefore, (18) appears to be a complete action which can successfully explain the history of cosmic evolution from the very early stage till date. Although, in view of our earlier discussion, we strongly believe that the presence of momentum constraints should not tell against the results obtained, it is of course important to check if it in fact does. However, it requires one to consider a more general metric consisting of $(0, i)$ components, where $i$ runs from 1 to 3 . This largely complicates the Hamiltonian and presently appears extremely difficult to handle. This issue may be addressed in the future.

Open Access This article is distributed under the terms of the Creative Commons Attribution 4.0 International License (http://creativecomm ons.org/licenses/by/4.0/), which permits unrestricted use, distribution, and reproduction in any medium, provided you give appropriate credit to the original author(s) and the source, provide a link to the Creative Commons license, and indicate if changes were made. Funded by $\mathrm{SCOAP}^{3}$.

\section{Appendix A: Matching the field equations}

It is now only left to prove that (31) is the correct Hamiltonian description of the theory (18) under consideration. This is important, since an auxiliary variable $Q$ (22) has been introduced to cast the action in canonical form, while no algebraic constraint has been added in the action to maintain the equivalence between the preceding (21) and following actions (23). However, it is enough to show that the Hamilton constraint equation (28) is equivalent to the $\left(\begin{array}{l}0 \\ 0\end{array}\right)$ component of Einstein's equation. This is because, replacing $p_{x}$ by $N Q$ and $x$ by $-\frac{p_{Q}}{N}$, the phase-space description of the Hamilton constraint equation (31) reduces to that presented in (30). Further, using the definition of momenta (22), one gets back the Hamiltonian (28).

Before we proceed, let us remember that the $\left(\begin{array}{l}0 \\ 0\end{array}\right)$ component of Einstein's equation, when multiplied by $\sqrt{-g}$, gives the Hamilton constraint equation. That is, if we start from the 
following Einstein-Hilbert action being minimally coupled to a scalar field,

$A=\int \sqrt{-g} \mathrm{~d}^{4} x\left[\frac{R}{2 \kappa}-\frac{1}{2} \phi_{, \mu} \phi^{, \mu}-V(\phi)\right]+\Sigma_{R}$,

then under metric variation, one obtains the field equation in the form

$\frac{G_{\mu \nu}}{\kappa}-T_{\mu \nu}=0$,

where $G_{\mu \nu}=\left(R_{\mu \nu}-\frac{1}{2} g_{\mu \nu} R\right)$ and $T_{\mu \nu}=\left(\nabla_{\mu} \phi \nabla_{\nu} \phi-\right.$ $\left.\frac{1}{2} g_{\mu \nu} \nabla_{\lambda} \phi \nabla^{\lambda} \phi-g_{\mu \nu} V\right)$ are the Einstein tensor and the energy-momentum tensor, respectively. The Hamiltonian is then

$H_{c}=\sqrt{-g}\left[\frac{G_{0}^{0}}{\kappa}-T_{0}^{0}\right]=0$.

This may be proved following the standard ADM (Arnwitt, Deser and Misner) formalism [70]. The ADM Hamiltonian is

$$
\begin{aligned}
H_{c}= & N\left[\frac{2 \kappa}{\sqrt{h}}\left(p^{i j} p_{i j}-\frac{1}{2} p^{2}\right)-\frac{\sqrt{h}^{(3)} R}{2 \kappa}+\frac{p_{\phi}^{2}}{\sqrt{h}}+\sqrt{h} V\right] \\
& -2 N_{i} p^{i j}{ }_{\| j}=N \mathcal{H}+N_{i} \mathcal{H}^{i},
\end{aligned}
$$

where $p^{i j}=-\frac{\sqrt{h}}{2 \kappa}\left(K^{i j}-K h^{i j}, p=\frac{\sqrt{h} K}{\kappa}\right.$ and $p_{\phi}=\frac{\sqrt{h}}{N} \phi_{, 0}$. $K^{i j}$ and $h^{i j}$ are the extrinsic curvature tensor and the three metric, respectively, while $K$ and $h$ are the corresponding traces. $N$ is the lapse function and $N_{i}$ is the shift vector. Further, $p^{i j}{ }_{\| j}$ is the covariant derivative of $p^{i j}$ in three space. Now in the background of Robertson-Walker metric (10), Eqs. (A3) and (A4) for the Hamiltonian lead to

$H_{c}=-\frac{3 a}{\kappa}\left(\frac{\dot{a}^{2}}{N}+k N\right)+a^{3}\left(\frac{\dot{\phi}^{2}}{2 N}+V N\right)=0$.

With the above understanding, let us start with the action (18), which under metric variation leads to the following field equation [89-92]:

$$
\begin{gathered}
\frac{G_{\mu \nu}}{\kappa}-T_{\mu \nu}+2 \beta\left(2 \xi(\phi) R R_{\mu \nu}+2 g_{\mu \nu} \square(\xi(\phi) R)\right. \\
\left.-2 \nabla_{\mu} \nabla_{\nu}(\xi(\phi) R)-\frac{1}{2} g_{\mu \nu} \xi(\phi) R^{2}\right)+2 \xi(\phi) H_{\mu \nu} \\
+8\left(\xi^{\prime 2} \nabla^{\rho} \phi \nabla^{\sigma} \phi+\xi^{\prime} \nabla^{\rho} \nabla^{\sigma} \phi\right) P_{\mu \rho \nu \sigma}=0,
\end{gathered}
$$

where

$$
\begin{aligned}
H_{\mu \nu}= & 2\left(R R_{\mu \nu}-2 R_{\mu \rho} R_{\nu}^{\rho}-2 R_{\mu \rho \nu \sigma} R^{\rho \sigma}\right. \\
& \left.+R_{\mu \rho \sigma \lambda} R_{\nu}^{\sigma \rho \lambda}\right)-\frac{1}{2} g_{\mu \nu} \mathcal{G}, \\
P_{\mu \nu \rho \sigma}= & R_{\mu \nu \rho \sigma}+2 g_{\mu[\sigma} R_{\rho] \nu}+2 g_{\nu[\rho} R_{\sigma] \mu}+R g_{\mu[\rho} g_{\sigma] \nu} .
\end{aligned}
$$

Now, in the background of the Robertson-Walker metric (10), the $\left(\begin{array}{l}0 \\ 0\end{array}\right)$ component of the field equation (A6) takes the following form:

$$
\begin{aligned}
& -\frac{36 \beta}{a^{2} N^{4}}\left(2 \dot{a} \dddot{a}-2 \dot{a}^{2} \frac{\ddot{N}}{N}-\ddot{a}^{2}-4 \dot{a} \ddot{a} \frac{\dot{N}}{N}+2 \dot{a}^{2} \frac{\ddot{a}}{a}+5 \dot{a} \frac{\dot{N}^{2}}{N^{2}}\right. \\
& \left.-2 \frac{\dot{a}^{3} \dot{N}}{a N}-3 \frac{\dot{a}^{4}}{a^{2}}-2 k N^{2} \frac{\dot{a}^{2}}{a^{2}}+\frac{k^{2} N^{4}}{a^{2}}\right) \\
& -\frac{72 \beta \xi^{\prime} \dot{a} \dot{\phi}}{a^{3} N^{4}}\left(a \ddot{a}+\dot{a}^{2}+k N^{2}-\frac{a \dot{a} \dot{N}}{N}\right)-\frac{3}{\kappa a^{2}}\left(\frac{\dot{a}^{2}}{N^{2}}+k\right) \\
& +\left(\frac{\dot{\phi}^{2}}{2 N^{2}}+V\right)-\frac{24 \xi^{\prime} \dot{\phi} \dot{a}}{N^{2} a^{3}}\left(\frac{\dot{a}^{2}}{N^{2}}+k\right)=0 .
\end{aligned}
$$

The Hamiltonian $H_{c}$ (28) on the other hand, may be expressed in terms of the scale factor $a$ as

$$
\begin{aligned}
& H_{C}=-\frac{36 \beta a}{N^{3}}\left(2 \dot{a} \dddot{a}-2 \dot{a}^{2} \frac{\ddot{N}}{N}-\ddot{a}^{2}-4 \dot{a} \ddot{a} \frac{\dot{N}}{N}+2 \dot{a}^{2} \frac{\ddot{a}}{a}\right. \\
& \left.+5 \dot{a} \frac{\dot{N}^{2}}{N^{2}}-2 \frac{\dot{a}^{3} \dot{N}}{a N}-3 \frac{\dot{a}^{4}}{a^{2}}-2 k N^{2} \frac{\dot{a}^{2}}{a^{2}}+\frac{k^{2} N^{4}}{a^{2}}\right) \\
& -\frac{72 \beta \xi^{\prime} \dot{a} \dot{\phi}}{N^{3}}\left(a \ddot{a}+\dot{a}^{2}+k N^{2}-\frac{a \dot{a} \dot{N}}{N}\right)-\frac{3 a}{\kappa}\left(\frac{\dot{a}^{2}}{N}+k N\right) \\
& +a^{3}\left(\frac{\dot{\phi}^{2}}{2 N}+V N\right)-\frac{24 \xi^{\prime} \dot{\phi} \dot{a}}{N}\left(\frac{\dot{a}^{2}}{N^{2}}+k\right)=0 .
\end{aligned}
$$

Clearly, multiplying Eq. (A8) by the same factor $\sqrt{-g}=$ $\mathrm{Na}^{3}$, the Hamiltonian (A9) is realized. Thus, the Hamiltonian obtained in the present methodology is the Hamiltonian of the action (18) under consideration. Now substituting $\mathcal{H}$ from Eq. (31), $p_{z}$ from Eq. (25b), $p_{\phi}$ from Eq. (25c), $p_{x}=N Q, x=$ $\frac{\dot{z}}{N}$ and $Q$ from Eq. (22), the canonical action (32) reduces to the action (21), which is essentially the action (18) in Robertson-Walker (10) minisuperspace.

\section{References}

1. A.G. Riess et al., Astron. J. 116, 1009 (1998)

2. S. Perlmutter et al., Astrophys. J. 517, 565 (1999)

3. E. Komatsu et al., Astrophys. J. Suppl. Ser. 192, 18 (2011)

4. D. Larson et al., Astrophys. J. Suppl. Ser. 192, 16 (2011)

5. S. Galli, R. Bean, A. Melchiorri, J. Silk, Phys. Rev. D 78, 063532 (2008)

6. D.N. Spergel et al., Astrophys. J. Suppl. Ser. 148, 175 (2003)

7. S. Nojiri, S.D. Odintsov, Phys. Rev. D 68, 123512 (2003)

8. S. Nojiri, S.D. Odintsov, Phys. Rep. 505, 59 (2011)

9. B. Modak, K. Sarkar, A.K. Sanyal, Astrophys. Space. Sci. 353, 407 (2014)

10. H. Weyl, Gravitation und Elektrizität. Sitzungsber. Preuss. Akad. d. Wiss. Teil 1, 465 (1918)

11. K. Stelle, Phys. Rev. D 16, 953 (1977)

12. T. Liko, Phys. Rev. D 77, 064004 (2008)

13. S. Sarkar, A.C. Wall, Phys. Rev. D 83, 124048 (2011) 
14. M.K. Parikh, Phys. Rev. D 84, 044048 (2011)

15. S. Chatterjee, M. Parikh, Class. Quant. Grav. 31, 155007 (2014)

16. M.H. Dehghani, S.H. Hendi, Int. J. Mod. Phys. D 16, 1829 (2007)

17. S.H. Hendi, Phys. Lett. B 677, 123 (2009)

18. S.H. Hendi, B.E. Panah, Phys. Lett. B 684, 77 (2010)

19. S.H. Hendi, S. Panahiyan, E. Mahmoudi, Eur. Phys. J. C 74, 3079 (2014)

20. C. Lanczos, Ann. Math. 39, 842 (1938)

21. D. Lovelock, J. Math. Phys. 12, 498 (1971)

22. Y. Choquet-Bruhat, J. Math. Phys. 29, 1891 (1988)

23. M. Brigante, H. Liu, R.C. Myers, S. Shenker, S. Yaida, Phys. Rev. D 77, 126006 (2008)

24. K. Izumi, Phys. Rev. D 90, 044037 (2014)

25. I. Antoniadis, J. Rizos, K. Tamvakis, Nucl. Phys. B 415, 497 (1994)

26. P. Kanti, J. Rizos, K. Tamvakis, Phys. Rev. D 59, 083512 (1999)

27. S. Kawai, J. Soda, Phys. Lett. B460, 41 (1999)

28. S. Kawai, M.A. Sakagami, J. Soda, Phys. Lett. B 437, 284 (1998)

29. R. Gregory, S. Kanno, J. Soda, JHEP 0910, 010 (2009)

30. Q. Pan, B. Wang, Phys. Lett. B 693, 159 (2010)

31. R.G. Cai, Z.Y. Nie, H.Q. Zhang, Phys. Rev. D 82, 066007 (2010)

32. J. Jing, L. Wang, Q. Pan, S. Chen, Phys. Rev. D 83, 066010 (2011)

33. Y.P. Hu, P. Sun, J.H. Zhang, Phys. Rev. D 83, 126003 (2011)

34. Y.P. Hu, H.F. Li, Z.Y. Nie, JHEP 01, 123 (2011)

35. A. Barrau, J. Grain, S.O. Alexeyev, Phys. Lett. B 584, 114 (2004)

36. N. Okada, S. Okada, Phys. Rev. D 79, 103528 (2009)

37. A.K. Sanyal, Phys. Lett. B 645, 1 (2007)

38. B.M. Leith, I.P. Neupane, JCAP 05, 019 (2007)

39. X.H. Ge, S.J. Sin, JHEP 05, 051 (2009)

40. R.G. Cai, Z.Y. Nie, N. Ohta, Y.W. Sun, Phys. Rev. D 79, 066004 (2009)

41. E.S. Fradkin, A.A. Tseytlin, Phys. Lett. B 163, 123 (1985)

42. J. Callan et al., Nucl. Phys. B 262, 593 (1985)

43. E. Bergshoeff, E. Sezgin, C.N. Pope, P.K. Townsend, Phys. Lett. B 188, $70(1987)$

44. D.J. Gross, J.H. Sloan, Nucl. Phys. B 291, 41 (1987)

45. R.R. Metsaev, A.A. Tseytlin, Phys. Lett. B 191, 354 (1987)

46. M.C. Bento, O. Bertolami, Phys. Lett. B 368, 198 (1996)

47. T. Torii, H. Yajima, K.I. Maeda, Phys. Rev. D 55, 739 (1997)

48. Z.K. Guo, N. Ohta, T. Torii, Prog. Theor. Phys. 120, 581 (2008)

49. Z.K. Guo, N. Ohta, T. Torii, Prog. Theor. Phys. 121, 253 (2009)

50. Z.K. Guo, N. Ohta, T. Torii, Prog. Theor. Phys. 121, 959 (2009)

51. B. Kleihaus, J. Kunz, E. Radu, Phys. Rev. Lett. 106, 151104 (2011)

52. I.P. Neupane, B.M.N. Carter, JCAP 0606, 004 (2006)

53. S. Nojiri, S.D. Odintsov, Phys. Lett. B 631, 1 (2005)

54. G. Cognola, E. Elizalde, S. Nojiri, S.D. Odintsov, S. Zerbini, Phys. Rev. D 73, 084007 (2006)

55. S. Tsujikawa, M. Sami, JCAP 0701, 006 (2007)

56. P. Binetruy, C. Charmousis, S.C. Davis, J.F. Dufaux, Phys. Lett. B 544, 183 (2002)

57. B.C. Paul, S. Ghose, Gen. Relativ. Gravit. 42, 795 (2010)
58. T. Koivisto, D.F. Mota, Phys. Lett. B 644, 104 (2007)

59. T. Koivisto, D.F. Mota, Phys. Rev. D 75, 023518 (2007)

60. A.K. Sanyal, Phys. Lett. B 81, 624 (2005)

61. A.K. Sanyal, Gen. Relativ. Gravit. 41, 1511 (2009)

62. A.K. Sanyal, C. Rubano, E. Piedipalumbo, Gen. Relativ. Gravit. 43, 2807 (2011)

63. S. Deser, J. Franklin, Class. Quant. Grav. 29, 072001 (2012)

64. T. Takahashi, J. Soda, Class. Quant. Grav. 29, 035008 (2012)

65. M. Henneaux, C. Teitelboim, J. Zanelli, Phys. Rev. A 36, 4417 (1987)

66. H.-H. Chi, H.-J. He, Nucl. Phys. B 885, 448 (2014)

67. E. Avraham, R. Brustein, Phys. Rev. D 90, 024003 (2014)

68. S. Ruz, R. Mandal, S. Debnath, A.K. Sanyal, Gen. Relativ. Gravit 48, 86 (2016)

69. K. Sarkar, N. Sk, R. Mandal, A.K. Sanyal, Int. J. Geom. Methods Mod. Phys. 14, 1750038 (2017). arXiv:1507.03444 [hep-th]

70. R. Arnowitt, S. Deser, C. Misner, Gravitation: An Introduction to Current Research (Wiley, New York, 1962)

71. A.K. Sanyal, S. Debnath, S.N. Ruz, Class. Quant. Grav. 29, 215007 (2012)

72. A.K. Sanyal, S. Debnath, S.N. Ruz, Phys. Rev. D 90, 047504 (2014)

73. A.K. Sanyal, Gen. Relativ. Gravit. 37, 1957 (2005)

74. G.T. Horowitz, Phys. Rev. D 31, 1169 (1985)

75. P. A. M. Dirac, Can. J. Math. 2, 129 (1950) [also in Lectures on Quantum Mechanics (Belfer Graduate School of Science, Yeshiva University, New York, 1964)]

76. J.W. York, Phys. Rev. Lett. 28, 1082 (1972)

77. G. Gibbons, S.W. Hawking, Phys. Rev. D 15, 2752 (1977)

78. E. Dyer, K. Hinterbichler, Phys. Rev. D 79, 024028 (2009)

79. M. Satoh, S. Kanno, J. Soda, Phys. Rev. D 77, 023526 (2008)

80. D.J. Schwarz, C.A. Terrero-Escalante, A.A. Garcia, Phys. Lett. B 517, 243 (2001)

81. S.M. Leach, A.R. Liddle, J. Martin, D.J. Schwarz, Phys. Rev. D 66, 023515 (2002)

82. D.J. Schwarz, C.A. Terrero-Escalante, JCAP 0408, 003 (2004)

83. M. Satoh, J. Soda, JCAP 0809, 019 (2008)

84. J.F. Dufaux, J.E. Lidsey, R. Maartens, M. Sami, Phys. Rev. D 70, 083525 (2004)

85. Z.K. Guo, D.J. Schwarz, Phys. Rev. D 81, 123520 (2010)

86. P.X. Jiang, J.W. Hu, Z.K. Guo, Phys. Rev. D 88, 123508 (2013)

87. M. Motaharfar, H.R. Sepangi, Eur. Phys. J. C 76, 646 (2016). arXiv: $1604.00453 \mathrm{v} 3$ [gr-qc]

88. D.G. Boulware, in Quantum Theory of Gravity, ed. by S.M. Christensen (Adam Hilger, Bristol, 1984)

89. Z. Guo, N. Ohta, T. Torii, Prog. Theo. Phys. 120, 581 (2008)

90. P. Kanti, B. Kleihaus, J. Kunz, Phys. Rev. D 85, 044007 (2012)

91. W. Ahn, B. Gwak, B. Lee, W. Lee, Eur. Phys. J. C 75(8), 372 (2015)

92. M. Ghodrati, Phys. Rev. D 90, 044055 (2014). arXiv:1404.5399 